\title{
Rapid cycling bipolar disorder post-heart transplant: A case report
}

\author{
Thompson $\mathrm{A}^{1,2}$, Cosker $\mathrm{G}^{2}$, Body $\mathrm{F}^{2}$, Hill $\mathrm{S}^{1}$ and Mukaetova-Ladinska EB $^{3 *}$ \\ ${ }^{1}$ Newcastle upon Tyne Hospitals NHS Foundation Trust, Newcastle upon Tyne, UK \\ ${ }^{2}$ Northumberland, Tyne and Wear NHS Foundation Trust, Newcastle upon Tyne, UK \\ ${ }^{3}$ Institute of Neuroscience, Psychology and Behaviour, University of Leicester, Leicester, UK
}

\begin{abstract}
There are few reports of psychiatric complications following transplant surgery in older people. Here we present a longitudinal assessment of a 69-year-old lady, a carrier of Duchenne's muscular dystrophy, who after having a heart transplant developed transient and prolonged neurological and psychiatric features. Initially the presentation resembled steroid induced psychosis with an underlying delirium; however, this evolved into an episode of rapid cycling diurnal mood variation. The following admission was due to being mute and un-cooperative with a poor oral intake. She was admitted to hospital again following a grand-mal seizure and now experiencing previous symptoms of delirium (hallucinations, irritability, and aggression) and had some cognitive impairment with fronto-executive dysfunction, alongside rapid cycling diurnal mood. We believe this to be the first time a rapid-cycling bipolar disorder has been described in an older person post-heart transplant surgery.
\end{abstract}

\section{Introduction}

Reports on psychiatric complications following transplant surgery are usually confined to younger people. In the case of heart transplants, posterior reversible encephalopathy syndrome (PRES) is well documented in both children [1] and adults [2], whereas reports about other psychiatric syndromes following heart transplant are rare. Depression [3-5], post-traumatic stress disorder, anxiety, adjustment disorder [6] and affective disorders [6,7] have all been documented within the first 3-6 years post-heart transplant with their prevalence being very high. Thus, up to $40 \%-51 \%$ of post-transplant patients have any of these psychiatric disorders [6,7]. In contrast, mania appears not be that frequent in this adult population, with only $8 \%$ having a manic episode post-transplant, and additional 33\% slightly elevated mood, highest within the first 3 weeks post-transplant [8]. Interestingly, the first onset of bipolar disorder has been documented as late as 5 years post-transplant [9].

However, the psychiatry of post-heart transplant (PHT) surgery in older people is not well defined. In the current case report we describe a longitudinal psychiatric assessment over a period of 4 years of a 69-year-old woman, a carrier of Duchenne's muscular dystrophy who developed prolong and transient episodes of rapid cycling bipolar disorder on a background of delirium with transient neurological features.

\section{Case report}

Mrs X, a 69-year-old carrier of Duchenne's muscular dystrophy, was initially referred to liaison psychiatry a few days following a heart transplant in 2013 when she was 65 years old. At that time, she had strong suicidal ideation and was aggressive towards family and medical/ nursing staff. Her brief postoperative hyponatraemia $(<125 \mathrm{mEq} / \mathrm{L})$ and hypocalcaemia $(<2 \mathrm{mmol} / \mathrm{L})$ resolved, but she continued to have labile mood and intermittent pressure of speech, was overtly sexually disinhibited and had auditory and visual hallucinations (seeing Elvis on the ceiling). The impression was of a steroid induced psychosis.

Whilst treated for delirium her corticosteroid therapy was adjusted, she developed a brief and transient episode of rapid cycling affective disorder, lasting a couple of weeks. Her CT brain scan showed extensive cortical atrophy with ventricular dilatation and leukoariosis. She was treated with Olanzapine and continued with the immunosuppressant therapy. Her mental state improved over the forthcoming 2 months, though she remained with poor oral intake and sleep. Intermittently, she had brief episodes of agitation and delusional beliefs of a grandiose nature accompanied by pressure of speech. Three months posttransplant her mental state was normalised, her MMSE was 30/30 and GDS 0/4. The MRI brain scan showed generalised cortical atrophy and ventricular dilatation in keeping with her age. She continued with immunosuppressant medication (tacrolimus and prednisolone) and was discharged.

Over the following period she was reviewed by the transplant team with no residual medical symptoms. However, 18 months post-surgery, she was again admitted to hospital due to cardiological problems and was re-started on corticosteroids. At this time, she also exhibited extrapyramidal neurological symptoms (i.e. tardive dyskinesia and lip smacking) of a few months duration, thought to be due to the small dose of olanzapine (2.5mg mane). Whilst an inpatient

*Correspondence to: Mukaetova-Ladinska EB, Institute of Neuroscience, Psychology and Behaviour, The University of Leicester, University Road, Leicester, UK, E-mail: eml12@le.ac.uk

Key words: heart transplant, rapid-cycling bipolar disorder, immunosuppressants Received: June 24, 2018; Accepted: July 11, 2018; Published: July 16, 2018 
she developed another episode of acute confusion, coinciding with increased dose of prednisolone where she was short of breath and anxious, rocking, staring at the same spot with fixed eyes for about 1 minutes and not responding. Her clinical presentation resembled both hypoactive delirium and depression, and she was started on a small dose of venlafaxine. A repeated CT head scan showed no new changes, whereas the MRI confirmed minor temporo-parietal vascular changes. A lumbar puncture revealed increased protein content $(0.67 \mathrm{~g} / \mathrm{l})$ but was negative for HSV1 and HSV2.

Three months later her physical and mental state improved, and she was discharged home with GP follow-up. However, 3 months after the discharge she was re-admitted again, now mute, with poor oral intake and uncooperative behaviour. Absences were noted during conversation where Mrs. X. would stop in the middle of the sentence and stare blankly. She would verbalise financial worries, repeating "there's nothing to live for" and "I'm dead". She was treated empirically for infection and discharged home.

After her last discharge, she apparently was well, and her husband had been caring for her at home. However, 7 months later, in July 2016, she was re-admitted again following a grand mal seizure, with confusion and agitation. A few weeks prior to this admission Mrs. X. had suffered a Bell's palsy, and this was followed with a rapid physical decline: she was unsteady on her feet and could not mobilise unaided, becoming increasingly un-co-operative, irritable, rude and using foul language. On admission, Mrs. X.'s significant weight loss was notable; she had an abundance of nihilistic delusions, was restless and crying out. Her NEWS score was ranging from 0-3 and she continued on subcutaneous fluids. NICE guidelines were followed, and she was treated with olanzapine for delirium. Except for EBV presence in her CSF, all other investigations were normal.

The symptomatology of her delirium episode appeared to have returned - she was again seeing and hearing 'Elvis', seeing images of her late parents but had no further episodes of agitation and irritation. Her working memory was significantly impaired. Suspicion at this time was of a steroid induced psychosis or encephalitis. Due to her uncooperativeness, all immunosuppressants were eventually stopped, and she continued to be treated according to the NICE guidelines for delirium. In the following month, she developed daily episodes of irritability and over familiarity, elated mood, hallucinations and aggression (i.e. hitting and even trying to stab nursing staff). During mornings she would remain unresponsive and not engaging. Her speech was incomprehensible and predominantly consisted of 'ah-ha, mmmm' and grunting sounds.

However, later in the day she would be over familiar, greeting staff with shouts and kisses and was very animated and impatient, and even physically and verbally abusive towards her family and staff. An ACE-R was 54/100, MMSE 19/30: she had global cognitive deficits with an element of fronto-executive dysfunction.

She continued to take orodispersible olanzapine which improved her appetite and she enjoyed spending most of her day away from the ward with her husband. She was eventually discharged and had no further contact with psychiatry services. According to her electronic notes she died a few months later, with no other information included.

\section{Clinical course}

- Initially referred with suicidal ideation, aggression, visual and auditory hallucinations, pressure of speech and sexual disinhibition: impression was of steroid induced psychosis.

- Corticosteroids reduced, delirium treated but episode of rapid cycling diurnal mood developed.

- Discharged from hospital after mental state normalised.

- Re-admitted 18 months later, rejecting her heart transplant and now back on high dose steroids.

- Experiencing extrapyramidal symptoms thought to be due to olanzapine.

- Developed acute confusion with high dose steroids, also had episodes similar to absence seizures.

- Treated for hypoactive delirium and depression.

- Clinical picture improved again and discharged home.

- 3 months later re-admitted mute, with poor oral intake and uncooperative. Pre-occupied by money and stating, 'I'm dead'. Treated empirically for infection and discharged after clinical presentation improved.

- Re-admitted 7 months later following a grand mal seizure, confused and agitated.

- Mobility now poor, reliant on a wheelchair and unable to mobilise independently.

- Treated for delirium after she appeared to be under the influence of hallucinations again.

- Immunosuppressants stopped due to patient being very uncooperative.

- Marked diurnal variation in presentation noted, subdued in the mornings but elated and somewhat grandiose in the afternoons.

- Cognitive assessments showed global cognitive deficits with fronto-executive dysfunction.

\section{Discussion}

The current case report draws the attention to the benefit of a longitudinal assessment by the same team over a period of time in an older person with a heart transplant. The psychiatric presentation of mixed co-existing features of both acute confusion and bipolar disorder complicate the diagnosis, and it was only after 4 years that the bipolar disorder became more evident.

Psychiatric symptoms are frequently reported in people PHT. Thus, in a study on 49 patients, anxiety (56\%) and mood disorders (60\% depressive episodes, $27 \%$ elevated mood) were most common and occurred within the first 3 weeks post-surgery [8]. Interestingly, even in this larger study, mania was relatively rare and occurred in only $3 / 49(6.12 \%)$ patients. Besides this report, to date there are 3 additional case reports on manic episodes post-transplant in younger people, occurring even 17 years post-transplant, of whom one had a previous diagnosis of bipolar disorder [10]. The case reported here is the first on an older person, with a rare genetic disease and no previous psychiatric history. Furthermore, we describe for the first time the development and re-occurrence of a rapid cycling bipolar disorder in 
later life, co-existing with longer manic and depressive episodes. Rapid cycling bipolar disorder has been previously described in one liver transplant patient [11], and although the course of the disorder seemed to be independent from any pharmacologic or psychosocial factor, the authors suggested that immunosuppressant's may have played some role in the pathogenesis of the syndrome.

Psychiatric and transient neurological symptoms have been described isolated in patients PHT. Factors increasing cumulative risk for psychiatric disorder post-transplant include pre-transplant psychiatric history, female gender, longer hospitalization, more impaired physical functional status, and lower social supports from caregiver(s) and family in the perioperative period [6]. In the case of Mrs. X., some of these risk factors were also present - female gender, longer hospitalisation and impaired physical functional status (i.e. impaired heart function, having delirium episodes). We cannot also exclude the possibility that the older age at the time of the transplant could have also played a role in the initiation of the overt mental health problems Mrs. X. had alongside her compromised physical state. However, a most recent study conducted on liver transplant patients demonstrated that age did not influence the extent of their anxiety and depression post-transplant [12]. This needs now to be confirmed in heart transplant patients.

Mrs. X.'s overall clinical presentation was accompanied by treatment-resistant medical and mental health features that needed longer term interventions and hospital admission. One of the reasons for this may be the organic nature of the psychiatric symptomatology, as confirmed via the CT and MRI brain scans: vascular post-transplant changes are commonly reported PHT. However, they may have a more significant impact on the clinical presentation in older people, who, as demonstrated in the current case presentation, may result in frontal encephalopathy. A recent study on 37 heart transplant patients similarly confirmed presence of cognitive impairment in nearly $40 \%$ of middle-age long-term survivors of heart transplant, with the majority having equivalent of mild cognitive impairment [13].

The transient neurological presentation in post-transplant heart patients has been attributed to side effects of immunosuppressant medication (including lowering seizure threshold) (reviewed in [14]). Since cerebrovascular disease and peripheral neuropathy are prevalent in patients with heart disease, the extrapyramidal neurological symptoms may be a direct consequence of cerebral vascular pathology. However, the extent of the vascular pathology and thus its accompanying neurological features may vary from patient to patient, and be more prominent in older people, further compromised with additional physical problems including anorexia, continuous heart inflammation and prolonged courses of immunosuppressant medication.

The continuous use of immunosuppressant therapy, especially high doses of corticosteroids, may have contributed to the initial presentation. However, we cannot exclude the possibility that they per sé may have precipitated further escalation of the manic symptoms 4 years post-transplant. In an earlier study on 73 patients, more than half of them had affective illness, predominantly steroid-related, and characterised with mood lability, irritability, and grandiosity [7]. In addition, continuous use of immunosuppressants alone, i.e. tacrolimus, can similarly underlie manic-like episodes, and they can be manifested even after 17 years post-transplant [15]. These data further support the polyaetiology of manic episodes in patients PHT. However, we have to highlight that the last episode of rapid cycling bipolar disorder that then evolved into a clear manic episode occurred when our patient was drug free for at least 1 month.
The longitudinal clinical evaluation of our patient suggests that mood changes, including elated affect, can easily be co-present in PHT patients with various degrees of severity. In older people PHT, we cannot exclude the possibility that the organic changes, restricted to the frontal lobe circuit, may modify the course of the psychiatric presentation, and result in longer, treatment resistant presentation. Although the PRES syndrome is well defined, the presence of manic episodes coexistent with cognitive dysfunction on a background of vascular pathology may also present as a minor clinical syndrome, namely the frontal PHT encephalopathy. Longitudinal multidisciplinary (i.e. psychiatric, neuroradiological, neuropsychological, neurological, biochemical investigations etc.) studies on PHT patients are now needed to define better this clinical syndrome.

\section{References}

1. Eilers B, Albers E, Law Y, McMullan DM, Shaw D, et al. (2016) Posterior reversible encephalopathy syndrome after paediatric heart transplantation: Increased risk for children with pre-existing Glenn/Fontan physiology. Pediatr Transplant 20: 552-558. [Crossref]

2. Dzudie A, Boissonnat P, Roussoulieres A, Cakmak, Mosbah K, et al. (2009) Cyclosporine-related posterior reversible encephalopathy syndrome after heart transplantation: Should we withdraw or reduce cyclosporine?: Case reports. Transplant Proc 41: 716-720. [Crossref]

3. Oswaldo Byrne L (1975) [A case of psychotic depression in a patient with a heart transplant]. Rev Neuropsiquiatr 38: 154-166. [Crossref]

4. Okwuosa I, Pumphrey D, Puthumana J, Brown RM, Cotts W (2014) Impact of identification and treatment of depression in heart transplant patients. Cardiovasc Psychiatry Neurol 2014: 747293. [Crossref]

5. Milaniak I, Wilczek-Rużyczka E, Wierzbicki K, Piatek J, Kędziora A, et al. (2018) The effect of clinical variables on distress and depressive symptoms among heart transplant recipients. Heart Lung 47: 68-72. [Crossref]

6. Dew MA, Kormos RL, DiMartini AF, Switzer GE, Schulberg HC et al. (2001) Prevalence and risk of depression and anxiety-related disorders during the first three years after heart transplantation. Psychosomatics 42: 300-313. [Crossref]

7. Shapiro PA, Kornfeld DS (1989) Psychiatric outcome of heart transplantation. Gen Hosp Psychiatry 11: 352-357. [Crossref]

8. Pudlo R, Miegza M,Zakliczynski M, Zembala M (2009) The occurrence of mood and anxiety disorders in heart transplant recipients. Transplant Proc 41: 3214-3218. [Crossref]

9. Hategan A, Nelson C, Jarmain S (2008) Heart transplant, social support, and psychiatric sequelae: a 10-year follow-up clinical case review. Psychosomatics 49: 3941. [Crossref]

10. Franić T, Kero T, Katić J, Marčinko D, Bilušić M (2012) Quetiapine in management of manic symptoms in patient with heart transplantation: a case report. Psychiatr Danub 24: 402-404. [Crossref]

11. Vieta E, de Pablo J, Cirera E, Grande L, Rimola A (1993) Rapidly cycling bipolar Il disorder following liver transplantation. Gen Hosp Psychiatry 15: 129-131. [Crossref]

12. Krenzien F, Krezdorn N, Morgul MH, Wiltberger G, Atanasov G, et al. (2017) The elderly liver transplant recipients: anxiety, depression, fatigue and life satisfaction. $Z$ Gastroenterol 55: 557-563. [Crossref]

13. Burker BS, Gude E, Gullestad L, Grov I, Relbo Authen A, et al. (2017) Cognitive function among long-term survivors of heart transplantation. Clin Transplant 31. [Crossref]

14. Heroux A, Pamboukian SV (2014) Neurologic aspects of heart transplantation. Handb Clin Neurol 121: 1229-1236. [Crossref]

15. Bersani G, Marino P, Valeriani G, Cuoco V, Zitelli C, et al. (2013) Manic-like psychosis associated with elevated trough tacrolimus blood concentrations 17 years after kidney transplant. Case Rep Psychiatry 2013: 926395. [Crossref]

Copyright: (C2018 Thompson A. This is an open-access article distributed under the terms of the Creative Commons Attribution License, which permits unrestricted use, distribution, and reproduction in any medium, provided the original author and source are credited. 